\title{
A Multifractal Random-Walk Description of Atmospheric Turbulence: Small-Scale Multiscaling, Long-Tail Distribution, and Intermittency
}

\author{
Lei $\mathrm{Liu}^{1} \cdot$ Fei $\mathrm{Hu}^{1} \cdot$ Shunxiang Huang ${ }^{2}$
}

Received: 12 September 2018 / Accepted: 9 April 2019 / Published online: 29 May 2019

(c) The Author(s) 2019

\begin{abstract}
The prevalent multifractal characteristics of turbulent velocity fluctuations in the atmosphere are important for estimating various wind effects in wind engineering. Here, the multifractal characteristics of turbulent velocity fluctuations, including the small-scale multiscaling, the long-tail distributions and the intermittency, are thoroughly investigated by using a highfrequency dataset of three-dimensional velocities $(100 \mathrm{~Hz})$ collected at three levels during one month. To reduce uncertainties in the estimate of multiscaling exponents, a new method, the sequential extended self-similarity, is proposed. Based on this method, we obtain the multiscaling exponents of $q$ th-order moments of velocity increments as a function of $q$, that is the so-called multifractal spectrum. The multifractal random walk (MRW) model is then shown to describe the various multifractal spectra of turbulent velocity fluctuations. With the help of this model, we find a connection between the small-scale multiscaling and the long-tail distributions, which is generally observed in our dataset, again validating the MRW model. A non-linear multifractal spectrum is commonly considered to be related to the intermittency of turbulent velocity fluctuations at small scales and its curvature is usually used as a quantification of intermittency intensity. However, we suggest that models capturing the nonlinear multifractal spectrum may fail to represent the long-tail distribution, which is a more direct quantification of intermittency. Finally, qualitative variations of validated indicators with specific boundary-layer parameters are investigated. Results show that the intermittency of turbulent velocity fluctuations is more relevant to the friction velocity, compared with the average wind speed, the average temperature, and the surface-layer stability.
\end{abstract}

Keywords Long-tail distribution · Multifractal random walk $\cdot$ Sequential extended self-similarity $\cdot$ Small-scale multiscaling $\cdot$ Turbulence intermittency

Fei Hu

hufei@mail.iap.ac.cn

Lei Liu

liulei@mail.iap.ac.cn

1 LAPC, Institute of Atmospheric Physics, Chinese Academy of Sciences, Beijing 100029, China

2 Institute of Chemical Defense, Beijing 102205, China 


\section{Introduction}

The small-scale multiscaling phenomenon has been found to be prevalent in the time series of turbulent velocity fluctuations in the atmosphere (Schmitt et al. 1994; Cho et al. 2001; Vindel and Yagüe 2011; Liu and Hu 2013; Xu and Hu 2015). Recently, studies have shown that small-scale multiscaling is important for estimating wind loads (Peinke et al. 2004; Fitton et al. 2014) and wind power (Milan et al. 2013; Calif and Schmitt 2014), and it is becoming a new feature in the statistical simulation of atmospheric turbulence (Nawroth and Peinke 2006; Guo et al. 2011; Calif and Schmitt 2012; Baile and Muzy 2016).

The turbulent velocity fluctuations $u(t)$ have small-scale multiscaling properties if the $q$ th-order moment of velocity increments,

$$
\left\langle|u(t+\tau)-u(t)|^{q}\right\rangle \sim \tau^{\zeta q},
$$

when the time scale $\tau$ is small enough and $\zeta_{q}$ is a non-linear function of $q$. The symbol $\langle\cdot\rangle$ stands for mathematical expectation. If $\zeta_{q}$ is linear, $u(t)$ becomes single-scaling. Multiscaling is a hallmark of multifractal, which refers to a class of geometrical objects displaying intermittency in time or space (Feder 1988; Mandelbrot et al. 1997).

In most statistical theories of turbulence, the small-scale multiscaling and intermittency are commonly attributed to the spatio-temporal intermittency of energy dissipation in the inertial range far from the energy-containing range (Frisch 1995). A multiscaling relation of velocity increments with a space lag of $l$,

$$
\left\langle|u(x+l)-u(l)|^{q}\right\rangle \sim l^{q / 3}\left\langle\varepsilon_{l}\right\rangle^{q / 3} \sim l^{q / 3+\theta_{q}},
$$

has been found to be true in laboratory turbulence (Meneveau and Sreenivasan 1991). It can be easily seen from this relation that if the energy dissipation rate $\varepsilon$ is not intermittent, its value averaged over a ball of size $l$, denoted by $\left\langle\varepsilon_{l}\right\rangle$, will not be related to $l$. Then $\theta_{q}$, which is a function of $q$, is zero and Eq. 2 is just the single-scaling result of the $\mathrm{K} 41$ model without intermittency (Kolmogorov 1941). According to Taylor's hypothesis, mean velocity can be used to translate turbulence measurements at a fixed spatial location over a time period to their corresponding measurements in space (Stull 1988). Thus, the above discussions about spatial multiscaling would also be true for the temporal multiscaling. Intermittency means that larger values occur more frequently than those predicted by the Gaussian distribution. Thus, many phenomenological models such as the log-normal model (Kolmogorov 1962), the log-gamma model (Saito 1992), the log- $\alpha$-stable model (Schmitt et al. 1992), and the log-Poisson model (She and Lévêque 1994) assume different long-tail distributions of energy dissipation to produce the non-linear multiscaling.

The variation of $\zeta_{q}$ with $q$ is generally considered to be universal for turbulence in the inertial range far from the energy-containing range. However, many previous studies on atmospheric turbulence have reported that the variation of $\zeta_{q}$ varies with large-scale factors such as stratification (Lauren et al. 1999; Shi et al. 2005; Vindel and Yagüe 2011; Cava et al. 2012; Wei et al. 2017), average wind speed (Böttcher et al. 2007; Vindel and Yagüe 2011), and surface roughness (Lauren et al. 1999; Katul et al. 2006, 2009; Cava et al. 2012). It should be noted that most observations of atmospheric turbulence focus on the low-frequency end of the inertial range. Thus, multiscaling at small scales in the inertial range close to the energycontaining range may be contaminated by large-scale motions (Katul et al. 1994; Mahrt 2014). This phenomenon is referred to as external intermittency, which is distinguished from so-called internal intermittency in the inertial range far from the energy-containing range. 
A united mathematical framework on small-scale multiscaling and the long-tail distribution is helpful for understanding, simulating and predicting the external intermittency of atmospheric turbulence. However, possibly due to the diversity of the external intermittency, this problem has been less systematically studied. Here, we note that the multifractal random walk (MRW) model can be used to resolve this problem (Muzy and Bacry 2002; Bacry and Muzy 2003). In this model, the small-scale multiscaling, the long-tail distribution, and the intermittency all emerge from a continuous construction of random multiplicative cascades. The MRW model formally unifies and extends results of turbulence phenomenological models listed above, which makes it suitable for describing the diversity of the external intermittency. Here, we mainly discuss whether the MRW model can be used to describe turbulent velocity fluctuations in the atmosphere. To achieve this goal, a high-frequency $(100 \mathrm{~Hz})$ dataset continuously collected during one month is used in our analyis. Statistical analysis always encounters difficulties due to limited samples with steady meteorological conditions, and the use of high-frequency data can improve results to a certain extent in comparison to the use of low-frequency data. In the following, we first briefly introduce the MRW model (Sect. 2) and then compare the multifractal spectrum (Sect. 4.2) and the probability density function (Sect. 5.1) of this model with data. A new method is also proposed for obtaining a more reliable multifractal spectrum at higher orders from data (Sect. 4.1). Finally, we define the intermittency exponent according to the MRW model (Sect. 5.2) and analyze the variations of the intermittency exponent with specific boundary-layer parameters (Sect. 5.3).

\section{Multifractal Random Walk}

We briefly review relevant characteristics of the multifractal random walk (MRW) model; for mathematical details, see Muzy and Bacry (2002) and references therein.

\subsection{Multifractal Random Walk Model}

The path of the MRW model $X(t)$, which is used to describe the velocity time series, can be built using two equivalent methods: (i) by subordinating a fractional Brownian motion $B_{H}(t)$ (Mandelbrot and Van Ness 1968; Feder 1988) with a multifractal random measure $M(t)$; (ii) by a stochastic integration of $M(t)$ against fractional Brownian noise. We use method (i) where $X(t)$ is given by

$$
X(t)=B_{H}[M(t)]
$$

The multifractal random measure $M(t)$ is a positive random process, and depending on the construction process of $M(t)$, the moments of $M(t)$ are either exact or asymptotic power functions of $t$. The asymptotically scaling moments of $M(t)$ are examined in order to investigate the multiscaling of turbulent velocity fluctuations at small scales. According to the characteristics of the multifractal random measure and the fractional Brownian motion, one can deduce that

$$
\left\langle|X(t)|^{q}\right\rangle \sim t^{\zeta q}, \text { if } t \rightarrow 0 .
$$

The function $\zeta_{q}$ is usually called the multifractal spectrum and can be derived from

$$
\zeta_{q}=q H-\varphi(-\mathrm{i} q H),
$$


where $H$ is a parameter that controls the statistical correlation behaviour of the fractional Brownian motion and $H \in(0,1)$ (Feder 1988). The function $\varphi(p)$ is the purely non-Gaussian Lévy-Khintchine variable (Sato 1999), that is

$$
\varphi(p)=\mathrm{i} m p+\int_{\mathbb{R}}\left(\mathrm{e}^{\mathrm{i} p x}-1-\mathrm{i} p \sin x\right) v(d x),
$$

where $v(d x)$ is a Lévy measure with $\int_{\mathbb{R}} \min \left(\left|x^{2}\right|, 1\right) v(d x)<\infty$ and $v(0)=0$, and $m \in \mathbb{R}$. Because $X(t)$ is assumed to have stationary increments, one can easily deduce that, if $\tau \rightarrow 0$,

$$
\left\langle|\delta X(\tau)|^{q}\right\rangle \sim \tau^{\zeta q}
$$

where $\delta X(\tau) \equiv X(t+\tau)-X(t)$.

\subsection{Multifractal Spectrum}

The multifractal spectrum $\zeta_{q}$ is determined by the Lévy measure $v(d x)$, and if $v(x)$ is known, $\zeta_{q}$ can be computed using Eqs. 5 and 6. In the following, we discuss three examples that are compared with observations.

(i) Deterministic MRW model. Let $v(d x)=0$, and we obtain

$$
\zeta_{q}=q H(1-m)
$$

For homogeneous and isotropic turbulence, $\left\langle|\delta u(\tau)|^{3}\right\rangle \approx\left|\left\langle\delta u(\tau)^{3}\right\rangle\right| \sim \tau$ (Frisch 1995; Benzi et al. 1993) and thus $\zeta_{3}=1$. In this case, $H(1-m)=1 / 3$ and $\zeta_{q}=q / 3$. One finds that this model recovers the K41 model only when $H(1-m)=1 / 3$ (Kolmogorov 1941).

(ii) Log-normal MRW model. Let $v(d x)=\mu \delta(x) d x / x^{2}$ with $\mu>0$, then $\varphi(-\mathrm{i} q)=$ $m q+\mu q^{2} / 2$. From Eq. 5, one has

$$
\zeta_{q}=q H-m q H-\mu \frac{q^{2} H^{2}}{2},
$$

and for homogeneous and isotropic turbulence with $\zeta_{3}=1$,

$$
\zeta_{q}=\frac{q}{3}-\mu \frac{H^{2} q(q-3)}{2} .
$$

When $H=1 / 3$, the above equation is equivalent to the multifractal spectrum of the lognormal model (Kolmogorov 1962).

(iii) Log-Poisson MRW model. Let $v(d x)=\gamma(\ln \beta)^{2} \delta(x-\ln \beta) d x / x^{2}$ with $\gamma>0$, then $\varphi(-\mathrm{i} q)=q[m-\gamma \sin (\ln \beta)]+\gamma\left(\beta^{q}-1\right)$. From Eq. 5, one has

$$
\zeta_{q}=q H[1-m+\gamma \sin (\ln \beta)]-\gamma\left(\beta^{q H}-1\right),
$$

and for homogeneous and isotropic turbulence with $\zeta_{3}=1$,

$$
\zeta_{q}=\frac{q}{3}\left[1+\gamma\left(\beta^{3 H}-1\right)\right]-\gamma\left(\beta^{q H}-1\right) .
$$

When $H=1 / 3, \gamma=2$ and $\beta=2 / 3$, Eq. 12 is equivalent to the multifractal spectrum of the log-Poisson model (She and Lévêque 1994). 


\subsection{Probability Density Function}

In this section, we list the main results on the probability density function (p.d.f.) of the MRW model; a detailed derivation can be found in the Appendix. The following equations are established with $t \rightarrow 0$ because $M(t)$ is built to be asymptotically multiscaling at small scales. The p.d.f. of the path of the MRW model at time $t$ is

$$
f_{t}(x)=\int_{0}^{\infty} g\left(\frac{x}{y}\right) w(\ln y) \frac{d y}{y^{2}},
$$

where $g(x)$ is the Gaussian distribution with zero mean and variance $\rho^{2}$. Function $w(x)$ is the p.d.f. of the random variable $\ln W_{t}^{H}$ whose characteristic function is

$$
\left\langle\mathrm{e}^{\mathrm{i} p \ln W_{t}^{H}}\right\rangle=t^{\mathrm{i} p H} \mathrm{e}^{-\varphi(p H) \ln t} .
$$

Note that the time $t$ above has been mapped to $[0,1]$ for convenience. Because of stationarity, if time $t$ in Eq. 13 is replaced by a time lag $\tau$ we obtain the p.d.f. of the increment of the MRW model over time $\tau$.

(i) Deterministic MRW model. In this case, $\varphi(p H)=\mathrm{imp} H$ and

$$
\left\langle\mathrm{e}^{\mathrm{i} p \ln W_{t}^{H}}\right\rangle=\mathrm{e}^{\mathrm{i} p \ln t^{(1-m) H}},
$$

showing that $W_{t}$ is deterministic and equals $t^{1-m}$. The deterministic MRW model is then proved to be Gaussian distributed with zero mean and variance $t^{2 H(1-m)} \rho^{2}$. It behaves like a fractional Brownian motion with a Hurst exponent of $H(1-m)$ if $H(1-m) \in(0,1)$.

(ii) Log-normal MRW model. In this case, $\varphi(p H)=\mathrm{imp} H-\mu p^{2} H^{2} / 2$ and

$$
\left\langle\mathrm{e}^{\mathrm{i} p \ln W_{t}^{H}}\right\rangle=\mathrm{e}^{\mathrm{i} p \ln t^{(1-m) H}+p^{2} \frac{\mu H^{2}}{2} \ln t},
$$

showing that $\ln W_{t}^{H}$ is Gaussian distributed with mean $\bar{\sigma}=(1-m) H \ln t$ and variance $\sigma^{2}=-\mu H^{2} \ln t$. According to Eq. 13, the p.d.f. of the log-normal MRW model is obtained by

$$
f_{t}(x)=\frac{1}{2 \pi \sigma \rho} \int_{0}^{\infty} \mathrm{e}^{-\frac{x^{2}}{2 \rho^{2} y^{2}}} \mathrm{e}^{-\frac{(\ln y-\bar{\sigma})^{2}}{2 \sigma^{2}}} \frac{d y}{y^{2}},
$$

similar to that proposed by Castaing et al. (1990). This type of p.d.f. has been widely used in the analysis of atmospheric turbulence (Beck et al. 2005; Böttcher et al. 2007; Laubrich and Kantz 2009; Liu and $\mathrm{Hu}$ 2013).

(iii) Log-Poisson MRW model. In this case, $\varphi(p H)=\mathrm{i} p H[m-\gamma \sin (\ln \beta)]+\gamma\left(\beta^{\mathrm{i} p H}-\right.$ 1) and

$$
\left\langle\mathrm{e}^{\mathrm{i} p \ln W_{t}^{H}}\right\rangle=\exp \left\{\mathrm{i} p H[1-m+\gamma \sin (\ln \beta)] \ln t-\gamma\left(\mathrm{e}^{\mathrm{i} p \ln \beta^{H}}-1\right) \ln t\right\} .
$$

This shows that $\ln W_{t}^{H}$ has the same distribution as a random variable $a \eta+b$ where $\eta$ is a Poisson distribution with mean $\lambda=\gamma(-\ln t)$ and

$$
\begin{aligned}
& a=\ln \beta^{H}, \\
& b=[1-m+\gamma \sin (\ln \beta)] H \ln t .
\end{aligned}
$$


According to Eq. 13, the p.d.f. of the log-Poisson MRW model is obtained from

$$
f_{t}(x)=\frac{1}{\sqrt{2 \pi} \rho} \sum_{k=0}^{\infty} \frac{1}{\mathrm{e}^{a k+b}} \exp \left(-\frac{x^{2}}{2 \rho^{2} \mathrm{e}^{2 a k+2 b}}\right) \frac{\lambda^{k} \mathrm{e}^{-\lambda}}{k !} .
$$

\section{Data}

Data were collected in August 2007 at a site located to the south-east of Dunhua $\left(43^{\circ} 12.643^{\prime} \mathrm{N}\right.$, $128^{\circ} 32.254^{\prime} \mathrm{E}$ ) in the north-east of China. The experimental site is surrounded by ridges that are about $100 \mathrm{~m}$ high in the east and south-west directions (see Fig. 1a). The surrounding terrain was a farmland planted with crops and soya beans, with height $\approx 1 \mathrm{~m}$. In the northwest direction, there is a village that located about $1.5 \mathrm{~km}$ from the experiment site (see Fig. 1b). Turbulence data including high-frequency velocity and temperature were collected using three ultrasonic anemometers (R3A-100, Gill, Lymington, UK) deployed at 2-m, 30-m and 100-m levels respectively on a 102-m tower (see Fig. 1c). The sampling frequency of the ultrasonic anemometer is $100 \mathrm{~Hz}$.

A double rotation method is used to transform the instrument reference frame to the streamline reference (Kaimal and Finnigan 1994). Herein, we focus on the wind speed $u$, computed from

$$
u=\sqrt{u_{l}^{2}+u_{v}^{2}+u_{w}^{2}},
$$

where $u_{l}, u_{v}$ and $u_{w}$ are velocity components in the longitudinal, lateral, and vertical directions after a double rotation. All boundary-layer parameters, such as the Obukhov length $L$ and the friction velocity $u_{*}$, are also computed using the rotated velocity components (Stull 1988). Data records are chronologically grouped into many samples each lasting $5 \mathrm{~min}$. The same analysis introduced in the following sections is repeated for each sample; samples with suspicious spikes and missing values are discarded with more than 7000 samples remaining. Unless otherwise noted, the averaging period is $5 \mathrm{~min}$ throughout.

\section{Small-Scale Multiscaling}

\subsection{Sequential Extended Self-Similarity}

Statistical moments of turbulence are found to obey the so-called extended self-similarity at a wide range of scales, even at large scales where the power-law scalings do not exist (Benzi et al. 1993). The mathematical expression of extended self-similarity is

$$
\left\langle|\delta u(\tau)|^{q}\right\rangle \sim\left\langle|\delta u(\tau)|^{p}\right\rangle^{\xi_{p, q}},
$$

and according to this expression, a better estimate of the multifractal spectrum $\zeta_{q}$ is achieved by avoiding to recognize scaling intervals artificially. For homogeneous and isotropic turbulence, $\left\langle|\delta u(\tau)|^{3}\right\rangle \sim \tau$ and the scaling exponent $\xi_{p, q}$ with $p=3$ equals $\zeta_{q}$. Thus, $\xi_{3, q}$ is commonly used to estimate the multifractal spectra of turbulent velocity fluctuations (Böttcher et al. 2007; Vindel and Yagüe 2011; Kiliyanpilakkil and Basu 2015).

Although sometimes $\xi_{3, q}$ is a good estimate of $\zeta_{q}$, it may perform poorly when $q$ is far from 3 . Besides, $\zeta_{3}$ is not always observed to be unity by analyzing turbulent data from normal 

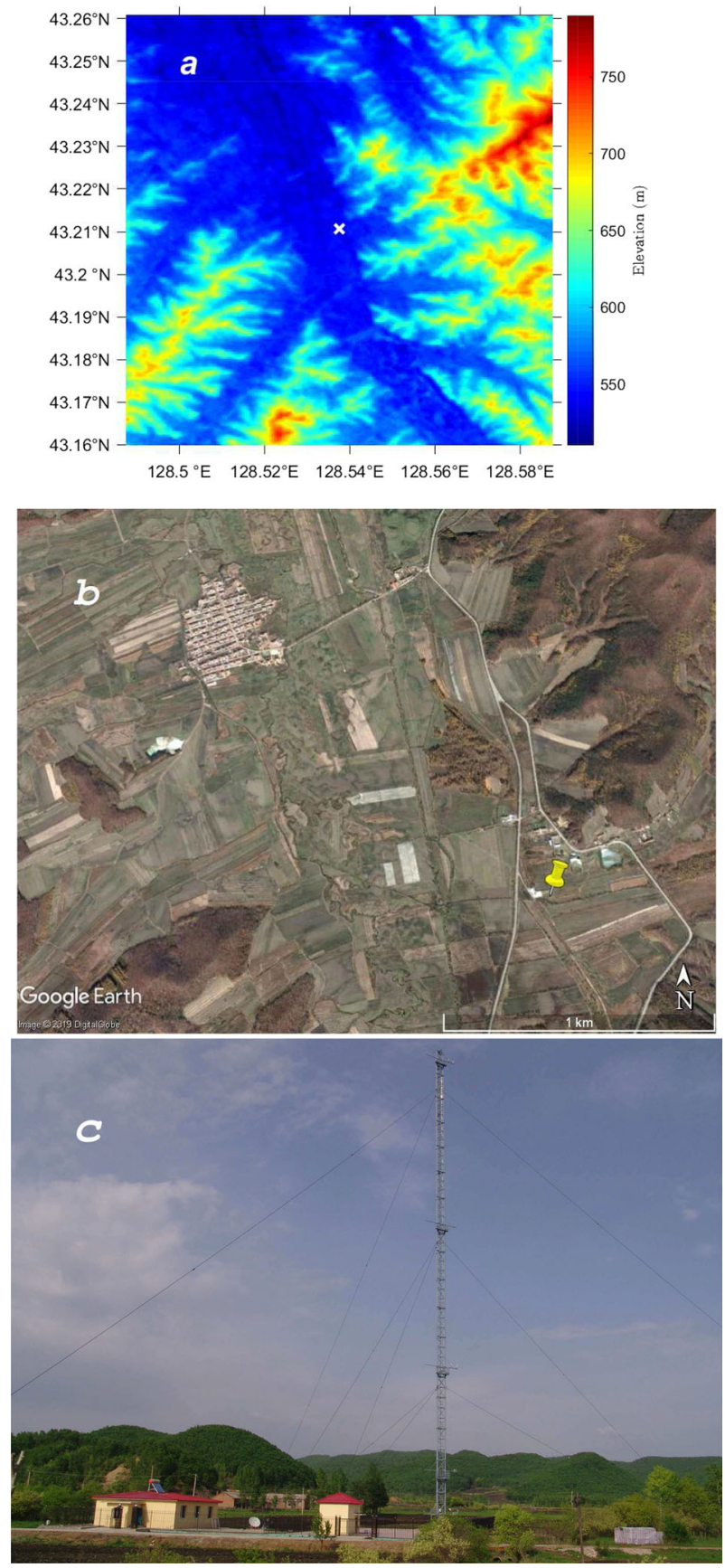

Fig. 1 a Elevation map around the experiment site (marked by a white cross). Data are from Version 2 of the ASTER Global Digital Elevation Model with a space resolution of 1 arc second (downloadable at https:// gdex.cr.usgs.gov/gdex/); b Google Earth image around the experiment site (marked by a yellow pushpin). c A photo of the 102-m tower. The surrounding houses were built for the experiment 
meteorological instruments such as the ultrasonic anemometer. The turbulence observational data may coexist with large-scale motions that are generally non-homogeneous and nonisotropic. In these cases, use of $\xi_{3, q}$ seems to be unreasonable. To improve the estimate of $\zeta_{q}$ especially with large values of $q$, we propose a new method that applies in more general cases. This method uses $\left\langle|\delta u(\tau)|^{q}\right\rangle$ versus $\left\langle|\delta u(\tau)|^{q-1}\right\rangle$ instead of versus $\left\langle|\delta u(\tau)|^{3}\right\rangle$, and call this method the sequential extended self-similarity. In this method, $\zeta_{q}$ is estimated by a recurrence relation $\zeta_{q}=\zeta_{q-1} \xi_{q-1, q}$, and if $\zeta_{1}$ is obtained by fitting $\ln \langle|\delta u(\tau)|\rangle$ versus $\ln \tau$ linearly, then

$$
\zeta_{q}=\zeta_{1} \xi_{1,2} \xi_{2,3} \cdots \xi_{q-1, q}
$$

The uncertainty in $\zeta_{q}$ is also estimated by a recurrence relation (Taylor 1997),

$$
\epsilon\left(\zeta_{q}\right)=\sqrt{\left[\zeta_{q-1} \epsilon\left(\xi_{q-1, q}\right)\right]^{2}+\left[\epsilon\left(\zeta_{q-1}\right) \xi_{q-1, q}\right]^{2}},
$$

where $\epsilon\left(\xi_{q-1, q}\right)$ denotes the estimation uncertainty of $\xi_{q-1, q}$ when fitting $\left\langle|\delta u(\tau)|^{q}\right\rangle$ versus $\left\langle|\delta u(\tau)|^{q-1}\right\rangle$. The estimation uncertainty is calculated from a $95 \%$ confidence bound.

Three methods to obtain $\zeta_{q}$ using a 5-min sample are illustrated in Fig. 2. The first method directly fits $\ln \left\langle|\delta u(\tau)|^{q}\right\rangle$ as a linear function of $\ln \tau$, where the fitting interval, where data nearly follow a line in the log-log plot, is usually recognized by eye. This procedure is shown in Fig. 2a. For simplicity, the upper boundaries of all fitting intervals are set to be $1 \mathrm{~s}$. In fact, with the increase of $q$, the artificial recognition of fitting intervals becomes more and more difficult. This would increase uncertainties of fittings (see squares in Fig. 2d). The second method fits $\ln \left\langle|\delta u(\tau)|^{q}\right\rangle$ as a linear function of $\ln \left\langle|\delta u(\tau)|^{3}\right\rangle$. This procedure is shown in Fig. 2b. When $q$ is not far from 3, one can see that the data follow a linear function almost in the whole interval of $\tau$. With the increase of $q$, the data become more fluctuating. The uncertainties of $\zeta_{q}$ will also increase with $q$ but will be generally smaller than those in the first method (see circles in Fig. 2d). The third method that is the sequential extended selfsimilarity fits $\ln \left\langle|\delta u(\tau)|^{q}\right\rangle$ as a linear function of $\ln \left\langle|\delta u(\tau)|^{3}\right\rangle$. The uncertainties of $\zeta_{q}$ are computed by Eq. 25. This procedure is shown in Fig. 2c. We found that even with large values of $q$ the data in the $\log -\log$ plot also vary linearly in the whole interval of $\tau$. Comparing with the first and second methods, we find that the uncertainties of $\zeta_{q}$ are further reduced by the third method (see triangles in Fig. 2d). More examples with different average wind speed $U$, average local temperature $T$, surface-layer stability $z / L$, and observation height $z$, are shown in Fig. 3. Symbols in each panel of Fig. 3 have the same meaning as in Fig. 2d. As shown in Fig. 3, the third method has the least uncertainties under most situations, and performs better than the commonly used first and second methods. We use the third method to obtain $\zeta_{q}$ in the following analysis.

\subsection{Comparison to Models}

Here the measured multifractal spectra $\zeta_{q}$ are compared with the spectra of the deterministic, log-normal and log-Poisson MRW models. Figure 3 shows four examples under various contrasted boundary-layer conditions (see Table 1): (i) smaller and larger average wind speeds with similar stabilities and average temperatures at the same observation height (see Fig. 3a, b); (ii) higher and lower average temperatures with similar stabilities and average wind speeds at the same observation height (see Fig. 3c, d); (iii) stable and unstable conditions with similar average wind speeds and temperatures at the same observation height (see Fig. 3e, f); (iv) lower and higher observation heights at the same observation time (see Fig. 3g, h). From the examples of (i), (ii) and (iii), one can see that the multifractal spectra $\zeta_{q}$ are likely to 
Fig. 2 Illustration of three methods to obtain the multifractal spectrum $\zeta_{q}$ of a 5-min sample of wind speeds. All fitting intervals in the first method are set to have the same upper bound (denoted by a broken line in panel a). Both the second (panel b) and the third method (panel c) fit data over the whole interval. For clarity, data points for $q>1$ have been shifted down arbitrarily. The coefficients of determination $R^{2}$ are also shown. The multifractal spectra obtained by the three methods are shown in panel $\mathbf{d}$. Uncertainties estimated by Eq. 25 are denoted by error bars in this panel
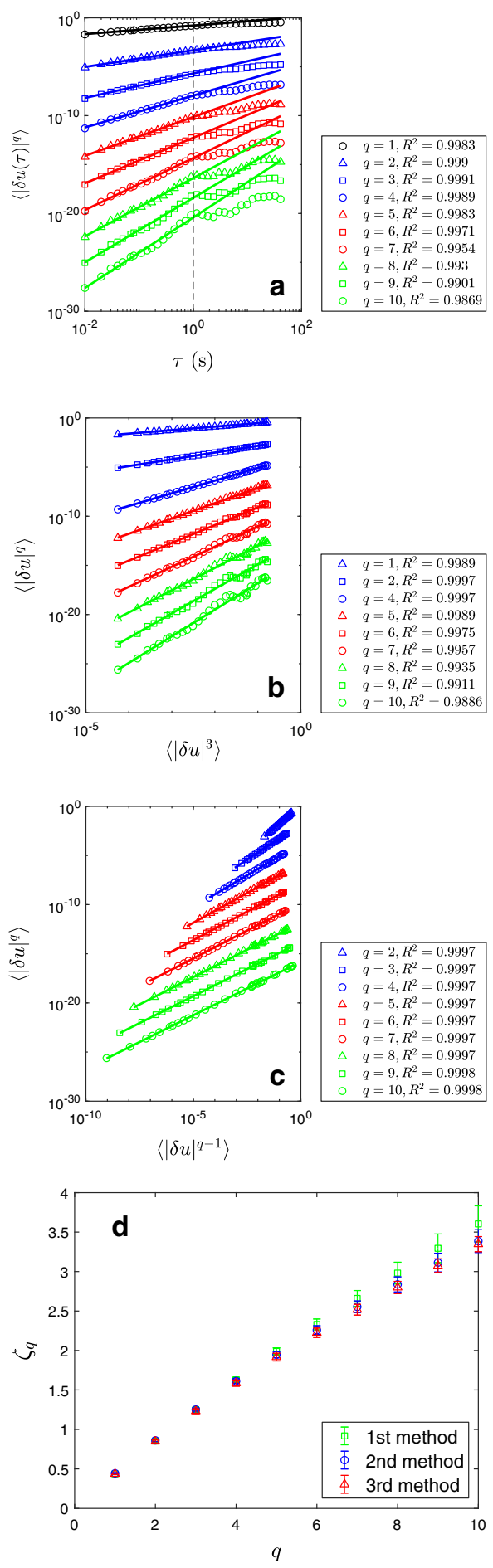

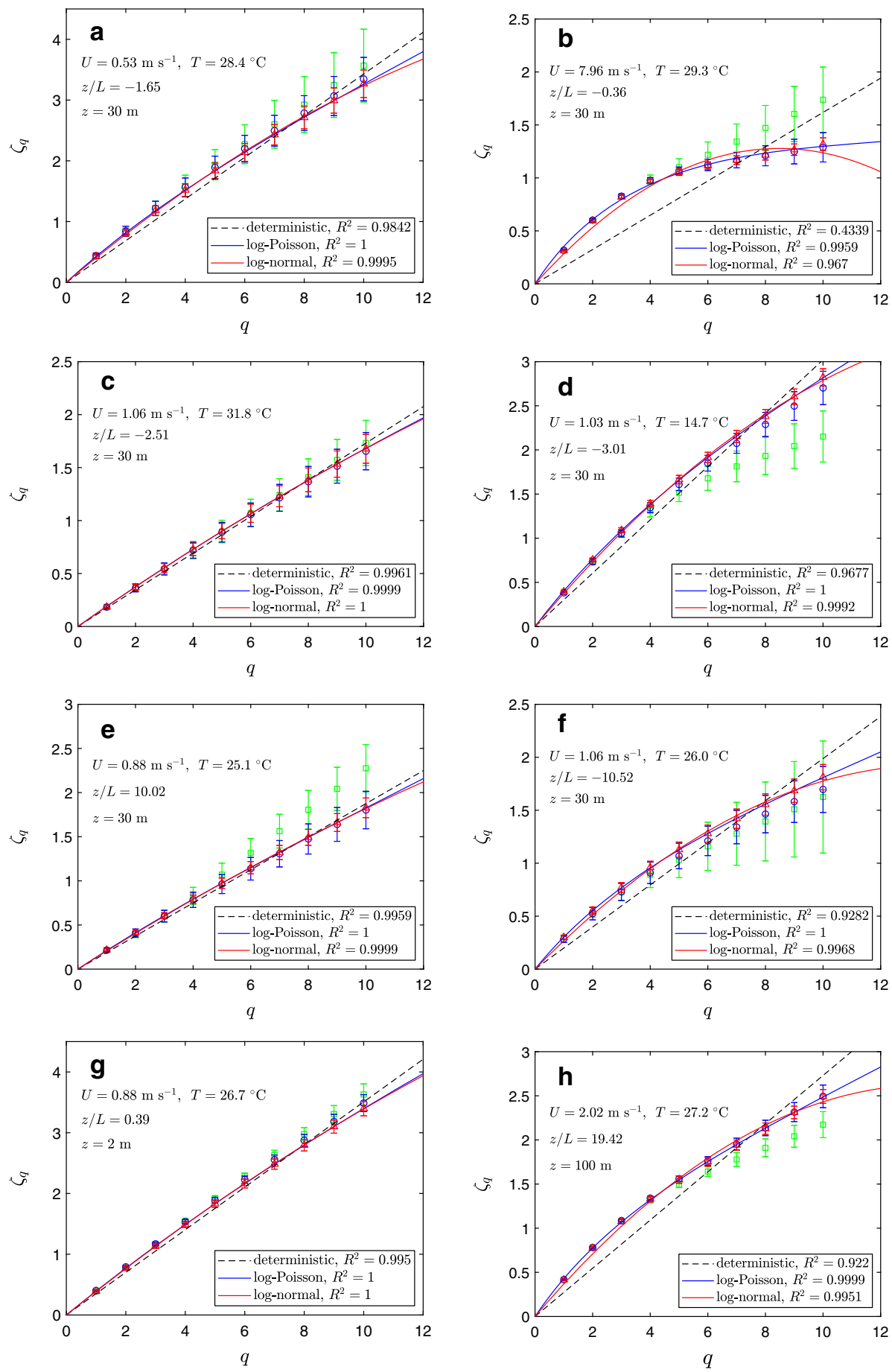

Fig. 3 Examples of the measured multifractal spectra and their comparisons with the multifractal spectra of the deterministic, the log-normal and the log-Poisson MRW models. Markers have the same meaning as in Fig. 2d 
Table 1 Flow characteristics of examples illustrated in Figs. 3, 4 and 5

\begin{tabular}{llllr}
\hline & $U\left(\mathrm{~m} \mathrm{~s}^{-1}\right)$ & $T\left({ }^{\circ} \mathrm{C}\right)$ & $z / L$ & $z(\mathrm{~m})$ \\
\hline Example (i) & & & & \\
$\quad$ Figures 3a, 4a & 0.53 & 28.4 & -1.65 & 30 \\
$\quad$ Figures 3b, 4b, 5a & 7.96 & 29.3 & -0.36 & 30 \\
$\quad$ Example (ii) & & & & \\
$\quad$ Figures 3c, 4c & 1.06 & 31.8 & -2.51 & 30 \\
$\quad$ Figures 3d, 4d & 1.03 & 14.7 & -3.01 & 30 \\
$\quad$ Example (iii) & & & & \\
$\quad$ Figures 3e, 4e & 0.88 & 25.1 & 10.02 & 30 \\
$\quad$ Figures 3f, 4f, 5b & 1.06 & 26.0 & -10.52 & 30 \\
$\quad$ Example (iv) & & & & \\
$\quad$ Figures 3g, 4g, 5c & 0.88 & 26.7 & 0.39 & 2 \\
$\quad$ Figures 3h, 4h, 5d & 2.02 & 27.2 & 19.42 & 100 \\
\hline
\end{tabular}

be more curved with a larger average wind speed and smaller average temperature in the unstable boundary layer. The example of (iv) shows that the $\zeta_{q}$ measured at higher height is more curved, and may be related to a larger average wind speed at a greater height. It should be noted that the more curved multifractal spectra may not necessarily correspond to more intermittent turbulent velocity fluctuations. The reason will be explained in the next section where the curvature of $\zeta_{q}$, the intermittency of atmospheric turbulence and their relations with boundary-layer parameters are discussed thoroughly in the framework of the MRW model.

From Fig. 3c, e and g, one can see that nearly linear multifractal spectra could be well fitted by the deterministic MRW model (black broken lines). These linear spectra can also be well fitted by the log-normal (red lines) and the log-Poisson MRW models (blue lines), because the two model both include the deterministic MRW model as their special cases. Besides, examples show that the two model spectra can also fit non-linear spectra and the lognormal MRW models fit data slightly better than the log-normal spectra. We have analyzed all samples and found that both the log-normal and the log-Poisson MRW models indeed fit the multifractal spectra of turbulent velocity fluctuations well under most situations.

\section{Long-Tail Distribution and Intermittency}

\subsection{Probability Density Functions At Different Scales}

In Sect. 2.3, we have derived the probability density functions (p.d.f.s) of the deterministic, the log-normal and the log-Poisson MRW models at different time scales. In this section, we compare these model p.d.f.s with data. From Eqs. 13 and 14, one can see that the p.d.f. for the MRW model at a fixed time scale is defined by a parameter $\rho$ and a function $\varphi(p H)$. The function $\varphi(p H)$ can be estimated by fitting the observed multifractal spectra with model data. This step has been discussed in the above section. The parameter $\rho$ can be obtained by the probability-of-return as follows. 
The probability-of-return for a symmetrical p.d.f. is defined by the probability density at the origin. Because the deterministic MRW model is Gaussian distributed, its probability-ofreturn is simply obtained from

$$
f_{\tau}(0)=\frac{1}{\sqrt{2 \pi} \rho} \tau^{-H(1-m)} .
$$

According to Eqs. 17 and 21, the probability-of-return is

$$
f_{\tau}(0)=\frac{1}{\sqrt{2 \pi} \rho} \tau^{-\mu H^{2} / 2-(1-m) H}
$$

for the log-normal MRW and is

$$
f_{\tau}(0)=\frac{1}{\sqrt{2 \pi} \rho} \tau^{-[1-m+\gamma \sin (\ln \beta)] H-\gamma\left(\beta^{-H}-1\right)}
$$

for the log-Poisson MRW model. Because the p.d.f. of the MRW model is derived at small scales, the corresponding Eqs. 26, 27 and 28 only hold when $\tau$ is small enough.

The probabilities-of-return of the three MRW models are all power functions. Parameters in the power exponents have been determined in the fitting processes of the multifractal spectra. The remaining unknown parameter $\rho$ can be obtained by fitting model probabilitiesof-return with data. In the data analysis, the probability defined on a small interval $(-h, h)$ can be used as an approximation of the probability-of-return. The examples used to illustrate the multifractal spectra in Fig. 3 are also used to illustrate the probabilities-of-return in Fig. 4. In the cases with nearly linear multifractal spectra (see Fig. 4c, g), one can see that the deterministic MRW model (black broken lines) can fit the data at small time scales as well as the log-normal MRW model (red lines) or the log-Poisson MRW model (blue lines). However, there are exceptions. As shown in Fig. 4e, any of the three models cannot fit the data, even the data behave as a power function over several orders of $\tau$ and the corresponding multifractal spectra are well fitted by models. In the non-linear cases, the log-Poisson MRW model would fit the data slightly better than the log-normal MRW model in general (see Fig. $4 \mathrm{a}, \mathrm{d}, \mathrm{f}$ and $\mathrm{h}$ ) but there are also exceptions (for example, see Fig. 4b).

Once the parameter $\rho$ and the function $\varphi(p H)$ are obtained, the model p.d.f.s are defined. We then compare the model p.d.f.s with observations. Before calculating p.d.f.s from observations, the velocity increments $\delta u(\tau)$ at the smallest time scale, that is $\tau=0.01 \mathrm{~s}$, are divided by their standard deviations. The p.d.f. at any scale is calculated by using these normalized data. In Fig. 5, some examples of p.d.f.s of velocity increments at scales $\tau=0.01 \mathrm{~s}$ (circles), $0.57 \mathrm{~s}$ (squares) and $40.96 \mathrm{~s}$ (triangles) are shown.

The left and right columns of Fig. 5 are comparisons of data with the p.d.f.s of the log-normal and the log-Poisson MRW models respectively. The Gaussian distributions at corresponding scales are also shown (broken lines in each panel). From this figure, one can see that p.d.f.s of velocity increments are generally symmetrical and go from non-Gaussian behaviour at smaller scales to Gaussian-like behaviour at larger scales. The non-Gaussian distributions at smaller scales have longer tails than the Gaussian distributions, which implies that at smaller scales large velocity fluctuations occur more frequently than is predicted from the Gaussian distribution and atmospheric turbulence at these scales would appear to be more intermittent than the Gaussian noise. Besides, one can see that the log-normal MRW model fits the tails of the observed p.d.f. much better than the log-Poisson MRW model at small scales (see Fig. 5a, b and d where the data correspond to those in Fig. 3b, f and h respectively). In linear cases (for example, see Fig. 5c in which the data correspond to those in Fig. 3g), both the log-normal and the log-Poisson MRW models can fit the data as well as the Gaussian 

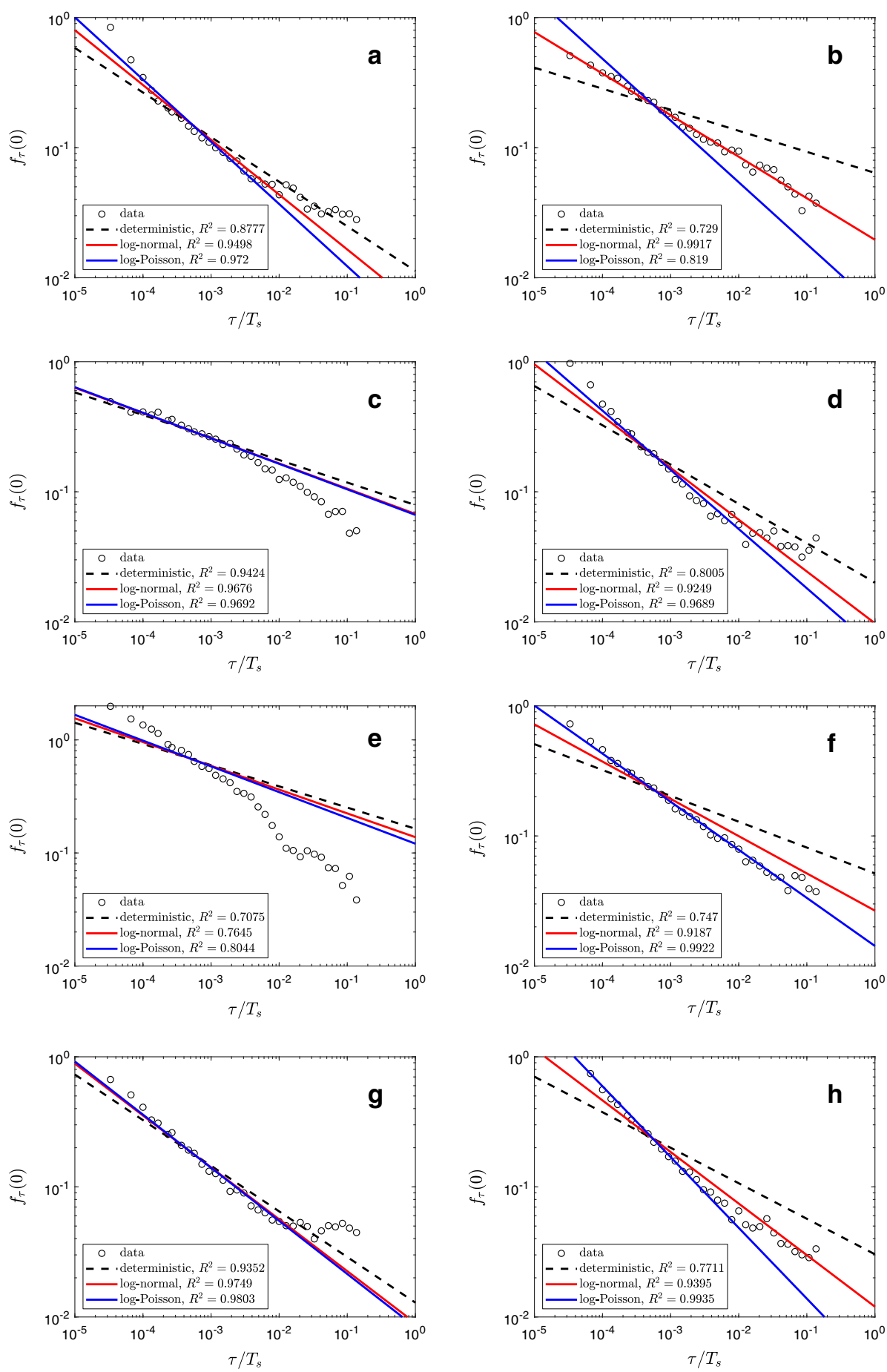

Fig. 4 Examples of probabilities of return and their comparisons with the MRW models. Note that the time scale $\tau$ has been normalized by the sample time $T_{S}$ of $5 \mathrm{~min}$. Data used in each panel are in one-to-one correspondence with those in Fig. 3 

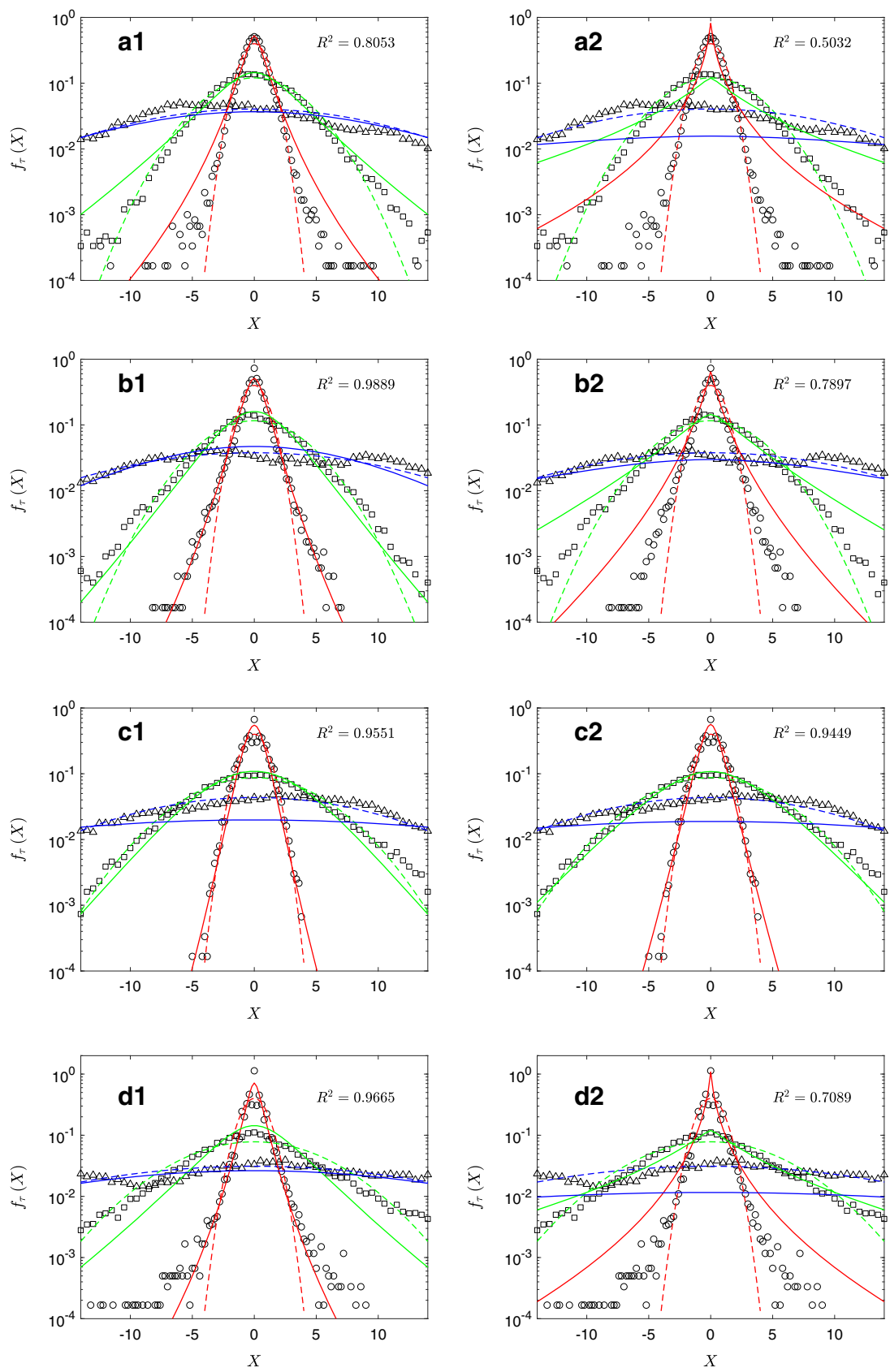

Fig. 5 Examples of p.d.f.s at time scales $\tau=0.01 \mathrm{~s}$ (circles), $0.57 \mathrm{~s}$ (squares) and $40.96 \mathrm{~s}$ (triangles) and their comparisons with the log-normal (left column) and the log-Poisson (right column) MRW models. Data used in panels a-d are in one-to-one correspondence with those in Fig. 3b, f, g and h respectively. Gaussian distributions with the same standard deviations of data are also shown for comparison (broken lines). Lines with different colours denote model fittings at $\tau=0.01 \mathrm{~s}$ (red), $0.57 \mathrm{~s}$ (green) and $40.96 \mathrm{~s}$ (blue) respectively. Note that velocity increments at $\tau=0.01 \mathrm{~s}$ have been normalized by their standard deviations and velocity increments at other scales are obtained by the normalized data. The determination coefficient $R^{2}$ at $\tau=0.01 \mathrm{~s}$ is also shown in each panel 
distributions. We have analyzed all samples and found that in general the log-normal MRW model indeed fits tails of the p.d.f. better than the log-Poisson MRW model, even the latter fits multifractal spectra slightly better.

\subsection{Intermittency Exponent}

In the above sections, we have shown that the log-normal MRW model is a good model to describe the small-scale multiscaling and the long-tail distributions of turbulent velocity fluctuations. Here, we revisit the issue of intermittency by using this model. In the classic log-normal model of isotropic and homogeneous turbulence (Kolmogorov 1962), the intermittency of velocities is attributed to the intermittency of dissipation. The idea is clearly manifest in a p.d.f. model of turbulent velocities proposed by Castaing et al. (1990). In this model, one can see that the only parameter, controlling p.d.f. tails of dissipation, also controls p.d.f. tails of turbulent velocities. With the increase of this parameter, p.d.f. tails become longer and longer and the corresponding time (or spatial) series of dissipation or velocities appear to be more and more intermittent. Because of this reason, this parameter, called the intermittency exponent, is commonly used to describe the intensity of turbulence intermittency. Although atmospheric turbulence is generally non-homogeneous and non-isotropic and its intermittency may not be attributed to the intermittency of dissipation, the intermittency exponent or its equivalent variants are also widely used to describe the intermittency of atmospheric turbulence (Vindel and Yagüe 2011).

When the multifractal spectrum of the log-normal MRW model formally collapses to that of the classic log-normal model, the parameter $\mu$ in the log-normal MRW model is just the intermittency exponent in the model of Castaing et al. (1990). Thus, the parameter $\mu$ is equivalent to this intermittency exponent. Although the use of parameter $\mu$ as an intermittency exponent is similar to much previous work, there is also a significant difference between our work and others. Many works estimated intermittency exponents only by fitting multifractal spectra, and did not test their estimations with observed p.d.f.s which are direct indicators of intermittency. We here put the estimated $\mu$ into the model of the log-normal MRW model and compare model p.d.f.s with observed p.d.f.s at the smallest scale. If deviation between observed and model p.d.f.s is large, the MRW model fails to describe data and the corresponding intermittency exponents, defined by this model, would be unreliable. The importance of the p.d.f. test is demonstrated in Fig. 6 where three time series of velocity increments at $\tau=0.01 \mathrm{~s}$ are shown as examples. Data used in Fig. 6a and b are the same as in Fig. 5a and c. The time series in Fig. 6a seems to be more intermittent than that in Fig. 6b, because the former has a lager intermittency exponent and longer p.d.f. tails (see Fig. 5a, c). The time series in Fig. $6 \mathrm{c}$ was obtained at the height of $100 \mathrm{~m}$. The corresponding flow characteristics are $U=6.43 \mathrm{~m} \mathrm{~s}^{-1}, z / L=-0.18$ and $T=30.1^{\circ} \mathrm{C}$. This series seems to be more intermittent than that in Fig. $6 \mathrm{~b}$ but has a smaller intermittency exponent. This is because the determination of coefficient of the p.d.f. of the time series in Fig. $6 \mathrm{c}$ is very small and the corresponding intermittency exponent fails to describe intermittency intensity.

\subsection{Variations of Intermittency Exponents With Friction Velocities, Wind Speeds, Temperatures, and Stabilities}

In this section, we analyze the variations of intermittency exponents $\mu$ with friction velocity $u_{*}$, local average wind speed $U$, local average temperature $T$, and surface-layer stability $z / L$. Before analyzing, we first test whether each 5-min sample can be described by the log- 


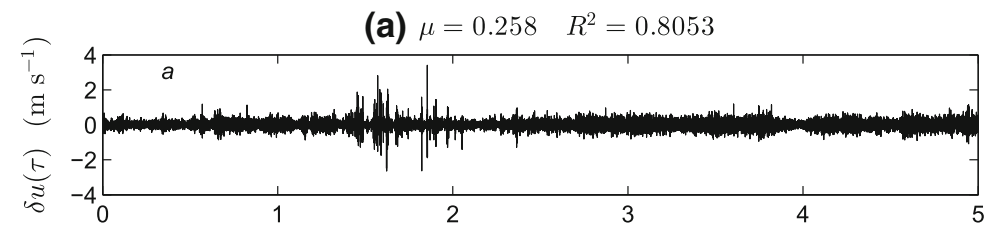

(b) $\mu=0.158 \quad R^{2}=0.9551$

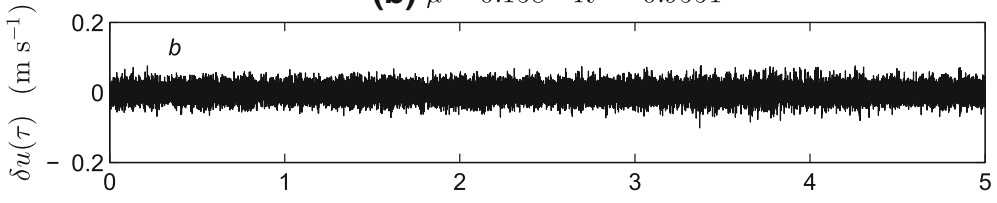

(c) $\mu=0.141 \quad R^{2}=0.08527$

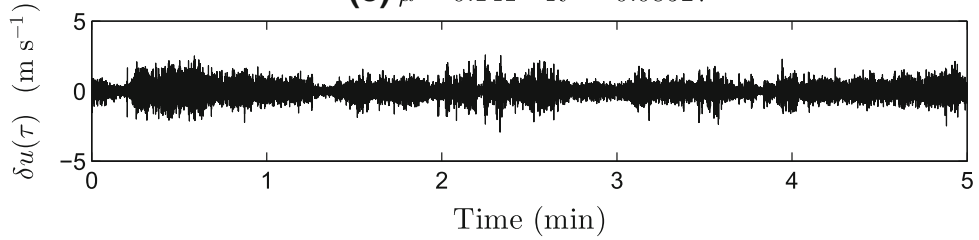

Fig. 6 Time series of velocity increments at $\tau=0.01 \mathrm{~s}$. The intermittency exponent $\mu$ and the corresponding determination coefficient $R^{2}$ of the p.d.f. fitting at $\tau=0.01 \mathrm{~s}$ are also shown in each panel

normal MRW model. If the determination of coefficient of its multifractal spectrum is greater than 0.99 or the determination of coefficient of its p.d.f. at the smallest scale $(\tau=0.01 \mathrm{~s})$ is greater than 0.80 , the sample is considered to be well described by the log-normal MRW model. Samples that do not satisfy this condition are discarded in the following analysis. At the end, about $82.5 \%, 76.3 \%$ and $74.4 \%$ samples at $2 \mathrm{~m}, 30 \mathrm{~m}$ and $100 \mathrm{~m}$ are left respectively. The corresponding numbers of remaining samples are 6417, 5934 and 5788.

The final results are shown in Fig. 7 where grey points represent the calculations of all 5-min samples and colour points represent the hourly-averaged values (black for 2-m data, blue for 30-m data and red for 100-m data). From Fig. 7a, one can see that if the friction velocity $u_{*}$ is very small, the corresponding intermittency exponent $\mu$ is also very small. With the increase of $u_{*}, \mu$ would quickly approach to the intermittency exponent of isotropic and homogeneous turbulence at the inertial range (denoted by broken lines). It means that the surface Reynolds stress may be a sensitive factor to the appearance of intermittency of turbulent velocity fluctuations. However, it may not be sensitive to the change of intermittency intensity if turbulent velocity fluctuations is already very intermittent. The variation of $\mu$ with $U$ is similar to that with $u_{*}$ but the former is more scattered (see Fig. 7b). Intermittency seems to be unrelated to the local temperature $T$ (see Fig. 7c) but seems to be related to the surfacelayer stability. It is found that large intermittency may appear more frequently in the unstable layer than in the stable one (see Fig. 7d), which means that the static stability may be a relevant factor to the change of intermittency intensity. Boundary-layer structures such as thermals in the unstable layer (Liu et al. 2012, 2014) and low-level jets, gravity waves or meandering motions in the stable layer (Mahrt 1999) possibly relate to the intermittency of turbulent velocity fluctuations. 

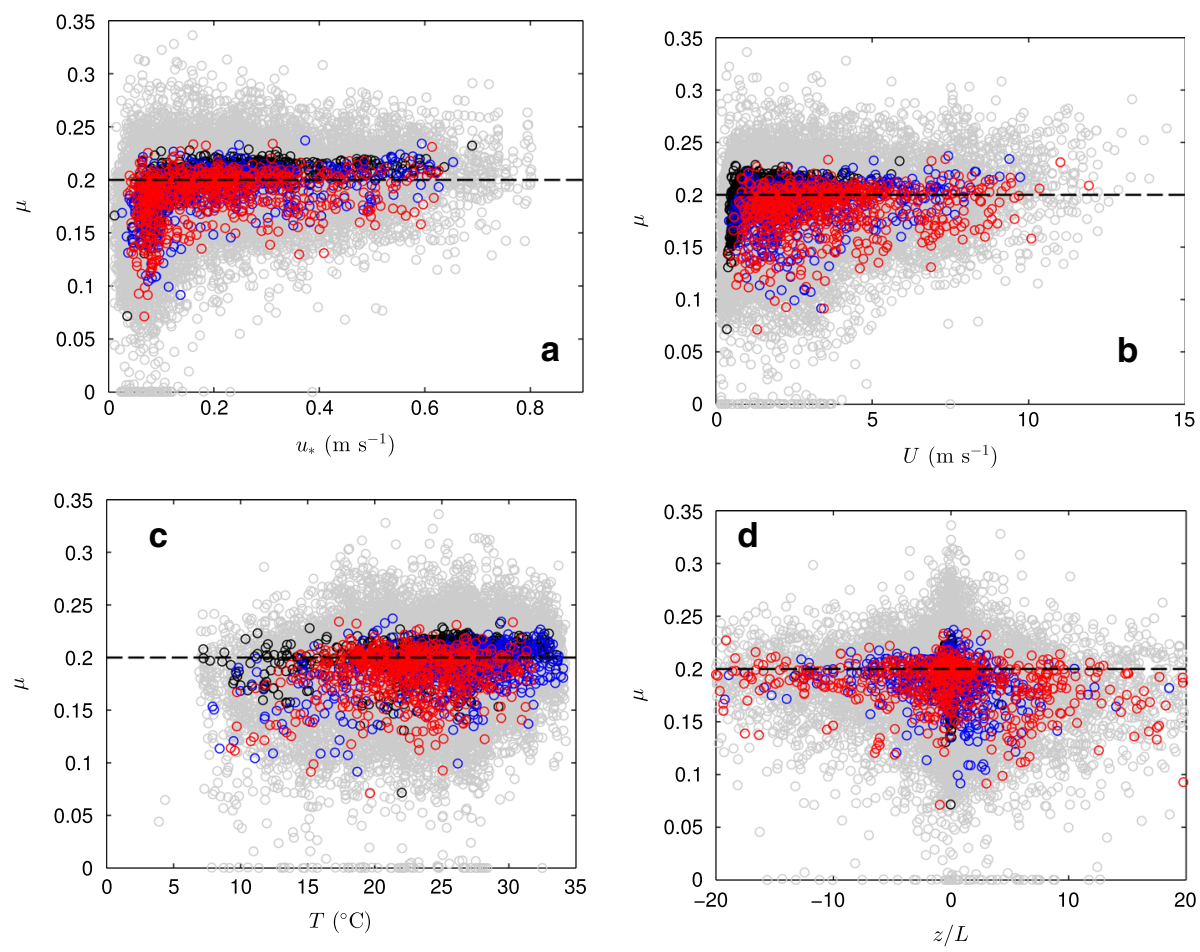

Fig. 7 Variations of intermittency exponents with friction velocity $u_{*}$, local average wind speed $U$, local average temperature $T$ and surface-layer stability $z / L$. The value of each grey point is computed from a 5-min sample. Colour points denote the hourly-averaged values of grey points at $z=2 \mathrm{~m}$ (black), $30 \mathrm{~m}$ (blue) and $100 \mathrm{~m}$ (red) respectively. The broken line in each panel denotes the intermittency exponent of homogeneous and isotropic turbulence at the inertial range

\section{Conclusions and Discussions}

We have used a high-frequency $(100 \mathrm{~Hz})$ velocity dataset continuously recorded for one month to analyze the intermittency of atmospheric turbulence. A new method, called the sequential extended self-similarity, is proposed to calculate the multifractal spectrum, i.e., the function of scaling exponent of $q$ th-order velocity moments at small scales with $q$. Results show that our method generally gives a better estimate than those commonly used. The multifractal spectrum is universal for homogeneous and isotropic turbulence but varies from case to case for atmospheric turbulence. The multifractal random walk (MRW) model, a mathematical model constructed by generalizing the random multiplicative cascade model, has a varied multifractal spectrum. This model is also found to have long-tail non-Gaussian distributions at small scales in general cases. We here compared the multifractal spectrum and the probability density function of the MRW model with atmospheric observations and found that the MRW model with a log-normal multifractal measure fits data well. Besides, our results suggest that the intermittency exponent, defined to describe the intermittency intensity and usually obtained from the multifractal spectrum, should be validated with the corresponding probability density function. It is because that long tails of a probability density function (compared with the tails of Gaussian distribution) are direct indicators of intermittency. If the probability density function of the MRW model with the same parameters 
as the corresponding multifractal spectrum is not fitted to data, the intermittency exponent estimated from the multifractal spectrum may be meaningless in describing the intermittency intensity. Finally, variations of validated intermittency exponents with boundary parameters are analyzed. Compared with the wind speed, the local temperature and the surface-layer stability, the intermittency exponent is found to be most relevant to the friction velocity. Large intermittency is also found to appear more frequently in the unstable surface layer than in the stable one.

Our work provides reliable statistical model of turbulent velocity fluctuations that would inspire new statistical simulation or forecasting methods in wind engineering. More importantly, we have raised some interesting problems. For homogeneous and isotropic turbulence, the multifractal measure in the MRW model is just the dissipation rate, but for the atmospheric turbulence, the intermittency is a kind of external intermittency and is possibly not mainly related to the dissipation rate. So, what is the physical meaning of the multifractal measure for atmospheric turbulence? Besides, what is the coherent structures of intermittency for atmospheric turbulence? Is there any other statistical indices that describe these coherent structures besides the intermittency exponents?

Acknowledgements This work is supported by the National Nature Science Foundation of China (Nos. 41675012 and 11472272) and the National Key Research and Development Program of China (Nos. 2016YFC0208802 and 2016YFC0209000). One of the authors Liu thanks his sons, Liu Xihe and Liu Xile. Without their cooperation, this paper would not be finished.

Open Access This article is distributed under the terms of the Creative Commons Attribution 4.0 International License (http://creativecommons.org/licenses/by/4.0/), which permits unrestricted use, distribution, and reproduction in any medium, provided you give appropriate credit to the original author(s) and the source, provide a link to the Creative Commons license, and indicate if changes were made.

\section{Appendix}

A mathematical derivation of the probability density function of the MRW model is presented here. According to the self-similarity of the fractional Brownian motion (Nourdin 2012), one has that $B_{H}(t) \stackrel{d}{=} \epsilon t^{H}$ where $\epsilon$ denotes a Gaussian random variable with zero mean and variance $\rho^{2}$. The symbol " $=$ " repersents equality in probability, and we have assumed that $B_{H}(0)=0$ without loss of generality. According to Eq. 3 in Sect. 2.1, one obtains

$$
X(t) \stackrel{d}{=} \epsilon M(t)^{H},
$$

where $\epsilon$ is independent of the multifractal random measure $M(t)$. Muzy and Bacry (2002) have proved that $M(t)$ follows a continuous cascade equation,

$$
M(\lambda t) \stackrel{d}{=} W_{\lambda} M(t)
$$

where $W_{\lambda}$ is a positive random variable independent of $M(t)$ and

$$
\begin{aligned}
& W_{\lambda}=\lambda \mathrm{e}^{\Omega \lambda}, \\
& \left\langle\mathrm{e}^{\mathrm{i} p \Omega \lambda}\right\rangle=\mathrm{e}^{-\varphi(p) \ln \lambda},
\end{aligned}
$$

with $\lambda \in(0,1]$. In the MRW model, $M(t)$ is generally assumed to be one in the whole time interval of a time series (Mandelbrot et al. 1997), implying that, if the time $t$ is mapped into $[0,1], M(1)=1$. Let $\lambda=t$ and $t=1$, one obtains

$$
X(t) \stackrel{d}{=} \epsilon W_{t}^{H}
$$


from Eqs. 29 and 30. According to Eqs. 33, 13 is then obtained. From Eq. 32, one can deduce that $\Omega_{t}$ is a infinitely divisible random variable (Sato 1999), and so $\ln W_{t}$ is also a infinitely divisible random variable and its characteristic function is

$$
\left\langle\mathrm{e}^{\mathrm{i} p \ln W_{t}}\right\rangle=t^{\mathrm{i} p}\left\langle\mathrm{e}^{\mathrm{i} p \Omega_{t}}\right\rangle=t^{\mathrm{i} p} \mathrm{e}^{-\varphi(p) \ln t} .
$$

Equation 14 is then obtained.

\section{References}

Bacry E, Muzy JF (2003) Log-infinitely divisible multifractal processes. Commun Math Phys 236:449-475

Baile R, Muzy JF (2016) Random cascade model for surface wind speed. In: Lehr JH, Keeley J (eds) Alternative energy and shale gas encyclopedia. Wiley, Hoboken, pp 153-162

Benzi R, Ciliberto S, Tripiccione R, Baudet C, Massaioli F, Succi S (1993) Extended self-similarity in turbulent flows. Phys Rev E 48:R29-R32

Beck C, Cohen EGD, Rizzo S (2005) Atmospheric turbulence and superstatistics. Europhys News 36:189-191

Böttcher F, St Barth, Peinke J (2007) Small and large scale fluctuations in atmospheric wind speeds. Stoch Environ Res Ris Assess 21:299-308

Calif R, Schmitt FG (2001) Modelling of atmospheric wind speed sequence using a lognormal continuous stochastic equation. J Wind Eng Ind Aerodyn 109:1-8

Calif R, Schmitt FG (2014) Multiscaling and joint multiscaling description of the atmospheric wind speed and the aggregate power output from a wind farm. Nonlinear Process Geophys 21:379-392

Castaing B, Gagne Y, Hopfinger EJ (1990) Velocity probability density functions of high Reynolds number turbulence. Phys D 46:177-200

Cava D, Katul GG, Molini A, Elefante C (2012) The role of surface characteristics on intermittency and zero-crossing properties of atmospheric turbulence. J Geophys Res 117:D01104

Cho JYN, Anderson BE, Barrick JDW, Thornhill KL (2001) Aircraft observations of boundary layer turbulence: intermittency and the cascade of energy and passive scalar variance. J Geophys Res 106:32469-32479

Feder J (1988) Fractals. Plenum, New York

Frisch U (1995) Turbulence. Cambridge University Press, Cambridge

Fitton G, Tchiguirinskaia I, Schertzer D, Lovejoy S (2014) Torque fluctuations in the framework of a multifractal 23/9-dimensional turbulence model. J Phys Conf Ser 555:012038

Guo H, Chen JJ, Qu QL, Liu PQ (2011) Generation of spatial atmospheric turbulence field in aircraft motion simulation based on refined similarity hypothesis. Sci China Technol Sci 54:244-250

Kolmogorov AN (1941) The local structure of turbulence in incompressible viscous fluid for very large Reynolds number. Dokl Akad Nauk SSSR 30:9-13

Kolmogorov AN (1962) A refinement of previous hypotheses concerning the local structure of turbulence in a viscous incompressible fluid at high Reynolds number. J Fluid Mech 13:82-85

Kaimal JC, Finnigan JJ (1994) Atmospheric boundary layer flows. Oxford University Press, New York

Katul GG, Parlange MB, Chu CR (1994) Intermittency, local isotropy, and non-Gaussian statistics in atmospheric surface layer turbulence. Phys Fluids 6:2480-2492

Katul GG, Porporato A, Cavac D, Siqueiraa M (2006) An analysis of intermittency, scaling, and surface renewal in atmospheric surface layer turbulence. Phys D 215:117-126

Katul GG, Porporato A, Poggi D (2009) Roughness effects on fine-scale anisotropy and anomalous scaling in atmospheric flows. Phys Fluids 21:035106

Kiliyanpilakkil VP, Basu S (2015) Extended self-similarity of atmospheric boundary layer wind fields in mesoscale regime: is it real? Europhys Lett 112:64003

Lauren MK, Menabde M, Seed AW, Austi GL (1999) Characterisation and simulation of the multiscaling properties of the energy-containing scales of horizontal surface-layer winds. Boundary-Layer Meteorol 90:21-46

Laubrich T, Kantz H (2009) Statistical analysis and stochastic modelling of boundary layer wind speed. Eur Phys J Spec Top 174:197-206

Liu L, Hu F (2013) Cascade-like and scaling behavior of wind velocity increments in the atmospheric surface layer. Phys A 392:5808-5816

Liu L, Hu F, Liu XM (2012) Proper orthogonal decomposition of mesoscale vertical velocity in the convective boundary layer. Boundary-Layer Meteorol 144:401-417

Liu L, Hu F, Cheng XL (2014) Extreme fluctuations of vertical velocity in the unstable atmospheric surface layer. Nonlinear Process Geophys 21:1-13 
Mahrt L (1999) Stratified atmospheric boundary layers. Boundary-Layer Meteorol 90:375-396

Mahrt L (2014) Stably stratified atmospheric boundary layers. Ann Rev Fluid Mech 46:23-45

Mandelbrot BB, Van Ness JW (1968) Fractional Brownian motions, fractional noises and applications. SIAM Rev 10:422-437

Mandelbrot BB, Fisher A, Calvet L (1997) A multifractal model of asset returns. Yale University, Cowles Foundation Discussion Paper \# 1164

Meneveau CM, Sreenivasan KR (1991) The multifractal nature of turbulent energy dissipation. J Fluid Mech 224:429-484

Muzy JF, Bacry E (2002) Multifractal stationary random measures and multifractal random walks with log infinitely divisible scaling laws. Phys Rev E 66:056121

Milan P, Wächter M, Peinke J (2013) Turbulent character of wind energy. Phys Rev Lett 110:138701

Nawroth AP, Peinke J (2006) Multiscale reconstruction of time series. Phys Lett A 360:234-237

Nourdin I (2012) Selected aspects of fractional Brownian motion. Springer, Italy

Peinke J, Barth S, Böttcher F, Heinemann D, Lange B (2004) Turbulence, a challenging problem for wind energy. Phys A 338:187-193

Saito Y (1992) Log-gamma distribution model of intermittency in turbulence. J Phys Soc Jpn 61:403-406

Sato K (1999) Lévy processes and infinitely divisible distributions. Cambridge University Press, Cambridge

Schmitt FG, Lavallée D, Schertzer D, Lovejoy S (1992) Empirical determination of universal multifractal exponents in turbulent velocity fields. Phys Rev Lett 68:305-308

Schmitt FG, Schertzer D, Lovejoy S, Brunet Y (1994) Empirical study of multifractal phase transitions in atmospheric turbulence. Nonlinear Process Geophys 1:95-104

She ZS, Lévêque E (1994) Universal scaling laws in fully developed turbulence. Phys Rev Lett 72:336-339

Stull RB (1988) An introduction to boundary layer meteorology. Kluwer academic publishers, Dordrecht

Shi B, Vidakovic B, Katul GG, Albertson JD (2005) Assessing the effects of atmospheric stability on the fine structure of surface layer turbulence using local and global multiscale approaches. Phys Fluids 17:055104

Taylor JR (1997) An Introduction to error analysis: the study of uncertainities in physical measurements, 2nd edn. University Science Books, Sausalito

Vindel JM, Yagüe C (2011) Intermittency of turbulence in the atmospheric boundary layer: scaling exponents and stratification Influence. Boundary-Layer Meteorol 140:73-85

Wei W, Wang MZ, Zhang HS, He Q, Ali M, Wang YJ (2017) Diurnal characteristics of turbulent intermittency in the Taklimakan desert. Meteorol Atmos Phys. https://doi.org/10.1007/s00703-017-0572-3

Xu JJ, Hu F (2015) Multifractal characteristics of intermittent turbulence in the urban canopy layer. Atmos Ocean Sci Lett 8:1-6

Publisher's Note Springer Nature remains neutral with regard to jurisdictional claims in published maps and institutional affiliations. 